\title{
SURVIVAL RATE, COMPLICATIONS AND PATIENT SATISFACTION WITH ZYGOMATIC \\ IMPLANTS: A REVISION OF THE LITERATURE
}

IMPLANT THERAPY OUTCOMES, SURGICAL ASPECTS
Moya-Villaescusa MJ, Terán-Casina G, Valdés-Beltrán A, Esteve-Colomina L.

Máster en Implantología y Rehabilitación Oral. Universidad Miguel Hernández. Elche, Alicante, España.

\section{Background and Aim}

Zygomatic implants (Zls) appeared at the late eighties to rehabilitate patients who had suffered resection of the maxilla. Nowadays, they are also used in patients with severe maxillary atrophy.

The stability of the $\mathrm{ZI}$ depends on the cortical bone it crosses during its placement.

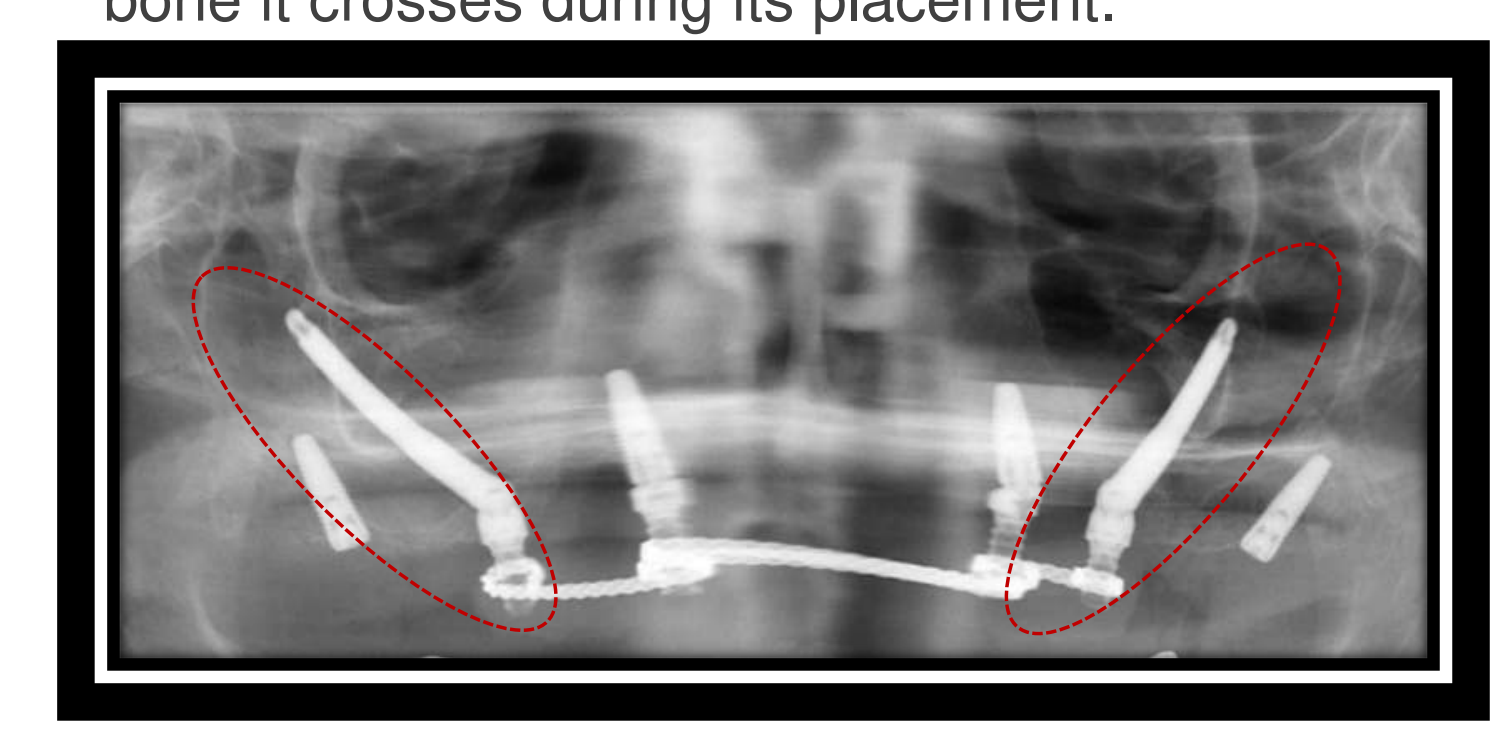

Aim: To assess the survival rate of Zls; the prevalence of complications and satisfaction level of the patients based on previously published studies with a medium- and long-term follow-up.

\section{Methods and Materials}

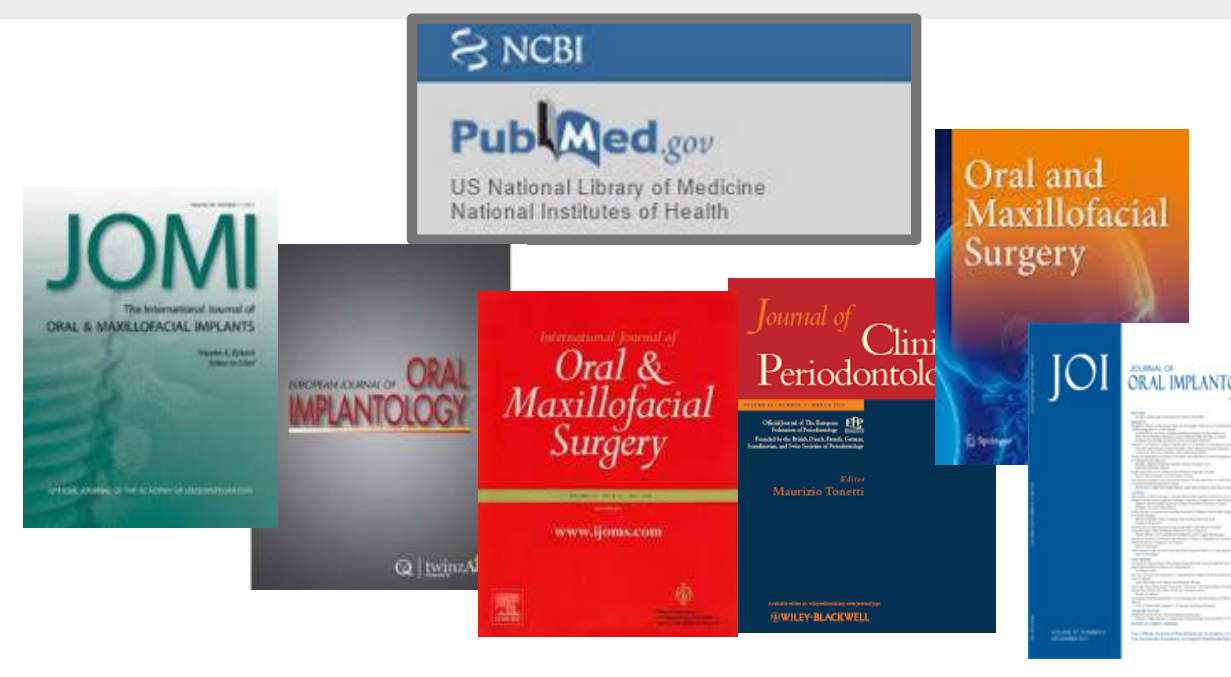

- An electronic search was performed in January/2017 in PubMed/Medline database and was supplemented by hand-searching.

- Search strategies :

\{Subject AND Adjective\}

\{Subject: (zygomatic OR zygoma OR zygomaticus)\}

- Adjective: (implant OR implants OR fixture OR fixtures)

\section{Exclusion criteria}

- Isolated cases.

- Animal studies.

- Review articles

- Inclusion criteria

Human studies receiving

$$
\text { Zis. }
$$

Minimum period of followup 39 months

.Variables extracted from these papers number of patients, number of zygomatic and standard implants (SI), duration of the study, surgical technique, type of prosthesis, type of loading, complications, survival rate of the Zls and satisfaction level of the patients

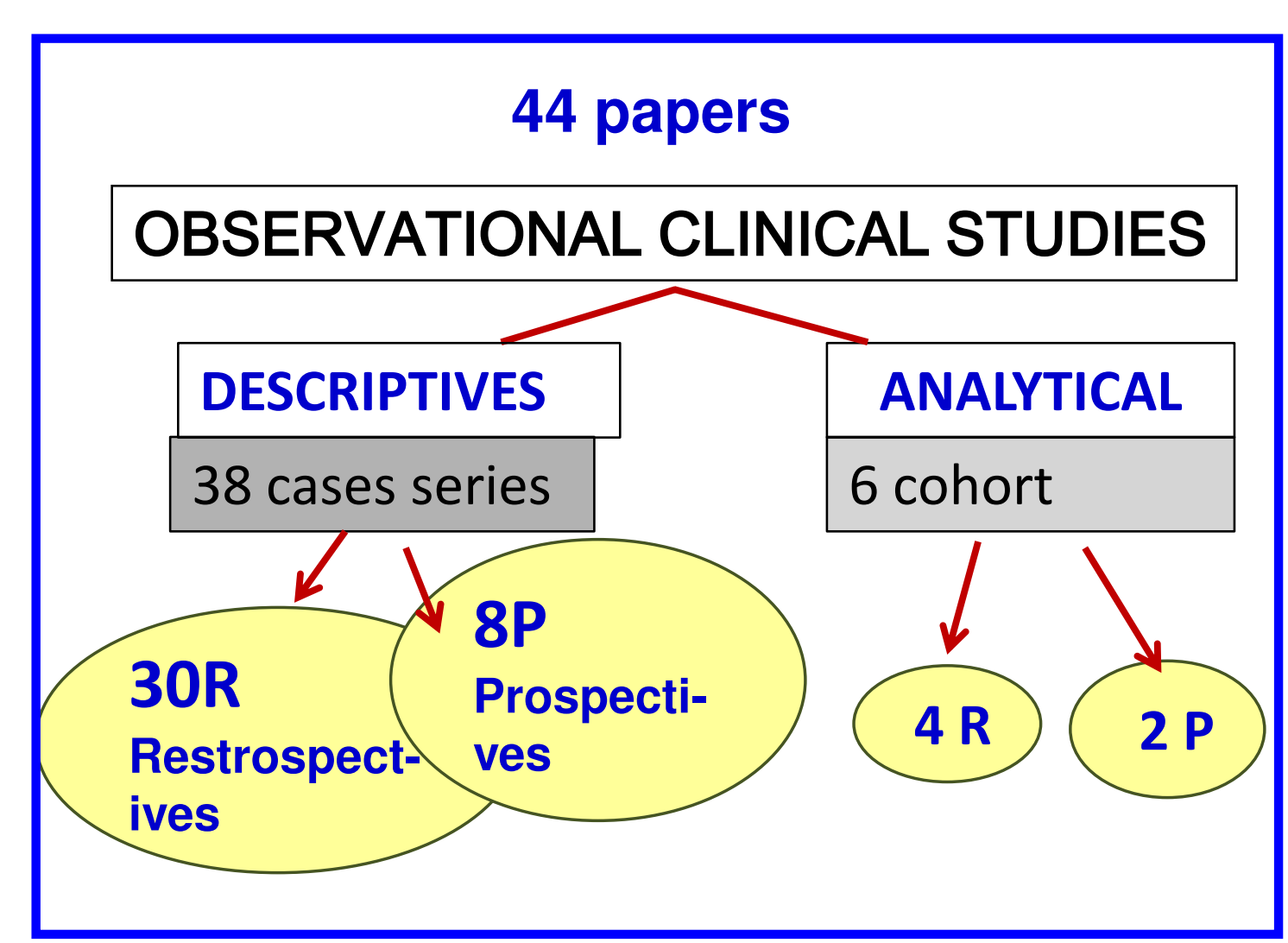

Results

\begin{tabular}{|c|c|c|c|}
\hline \multicolumn{3}{|c|}{ STUDY SAMPLE AND SURVIVAL RATE } & S| \\
\hline TOTAL & 1638 & 3589 & 4117 \\
\hline № failures & & 90 & 138 \\
\hline $\begin{array}{l}\text { global survival } \\
\text { rate (\%) }\end{array}$ & & 97,5 & 96,65 \\
\hline $\begin{array}{l}39-120 \\
\text { months }\end{array}$ & 1336 & 2943 & 3711 \\
\hline № failures & & 64 & 93 \\
\hline $\begin{array}{l}\text { survival rate } \\
(\%)\end{array}$ & & 97,82 & 97,5 \\
\hline$>120$ months & 302 & 646 & \\
\hline № failures & & 26 & 45 \\
\hline $\begin{array}{l}\text { Survival rate } \\
(\%)\end{array}$ & & 95,97 & 88,9 \\
\hline
\end{tabular}

ZI: zygomatic implantes; SI: standard implants

SURVIVAL RATE ACCORDING TO SURGICAL TECHNIQUE

\begin{tabular}{|c|c|c|c|}
\hline $\begin{array}{c}\text { Surgical } \\
\text { techique }\end{array}$ & Patients & ZI & $\begin{array}{c}\text { № } \\
\text { papers }\end{array}$ \\
\hline
\end{tabular}

\begin{tabular}{|l|c|c|c|}
\hline CT & 806 & 1710 & 24 \\
\hline Failures/ & & 41 & \\
\hline
\end{tabular}

\begin{tabular}{|l|l|r|r|}
\hline SS & 144 & 341 & 6 \\
\hline
\end{tabular}

Failures/ $\quad 17(4,9 \%) / 95,1 \%$

survival

\begin{tabular}{|l|c|c|c|}
\hline ET & 436 & 942 & 6 \\
\hline Failures/ & & $11(1,16 \%) /$ & \\
\hline survival & & $98,84 \%$ & \\
\hline GS & & & \\
\hline
\end{tabular}

GS

10

Failures/

survival

\begin{tabular}{|l|l|l|l|}
\hline ZAGA & 80 & 157 & 1 \\
\hline
\end{tabular}

\begin{tabular}{l|c} 
Failures/ & $50(3,1 \%) / 96,9 \%$
\end{tabular}

survival

MZI

Failures/

survival

CT: clasical technique; $\mathbf{S S}$ : sinus slot; ET: extrasinusal technique; CS: guide surgery; ZAGA: Zygoma Anatomy Guided Approach; MZI: multiple zygomatic Anatomy
implants.

\begin{tabular}{|l|c|c|c|}
\hline \multicolumn{4}{|c|}{ SURVIVAL RATE ACCORDING TO LOADING PROTOCOL } \\
\hline \begin{tabular}{l}
\multicolumn{1}{|c|}{ Loading } \\
protocol
\end{tabular} & Patients & ZI & № papers \\
\hline $\begin{array}{l}\text { Deferred (4- } \\
\text { 6 months) }\end{array}$ & 685 & 1500 & 28 \\
\hline $\begin{array}{l}\text { Failures/ } \\
\text { survival }\end{array}$ & & $\begin{array}{c}43(2,8 \%) / \\
97,2 \%\end{array}$ & \\
$\begin{array}{l}\text { Early } \\
\text { (8 weeks) }\end{array}$ & 20 & 40 & 1 \\
\hline $\begin{array}{l}\text { Failures/ } \\
\text { survival }\end{array}$ & & $\begin{array}{c}0(0 \%) / \\
100 \%\end{array}$ & \\
Immediate & 933 & 2049 & 17 \\
\hline $\begin{array}{l}\text { Failures/ } \\
\text { survival }\end{array}$ & & $\begin{array}{c}144(2,14 \%) \\
/ 97,86 \%\end{array}$ & \\
\hline
\end{tabular}

\begin{tabular}{|c|c|}
\hline PROSTHETIC COMPLICATIONS ACCORDING THE \\
SURGICAL TECHNIQUE
\end{tabular}

Results

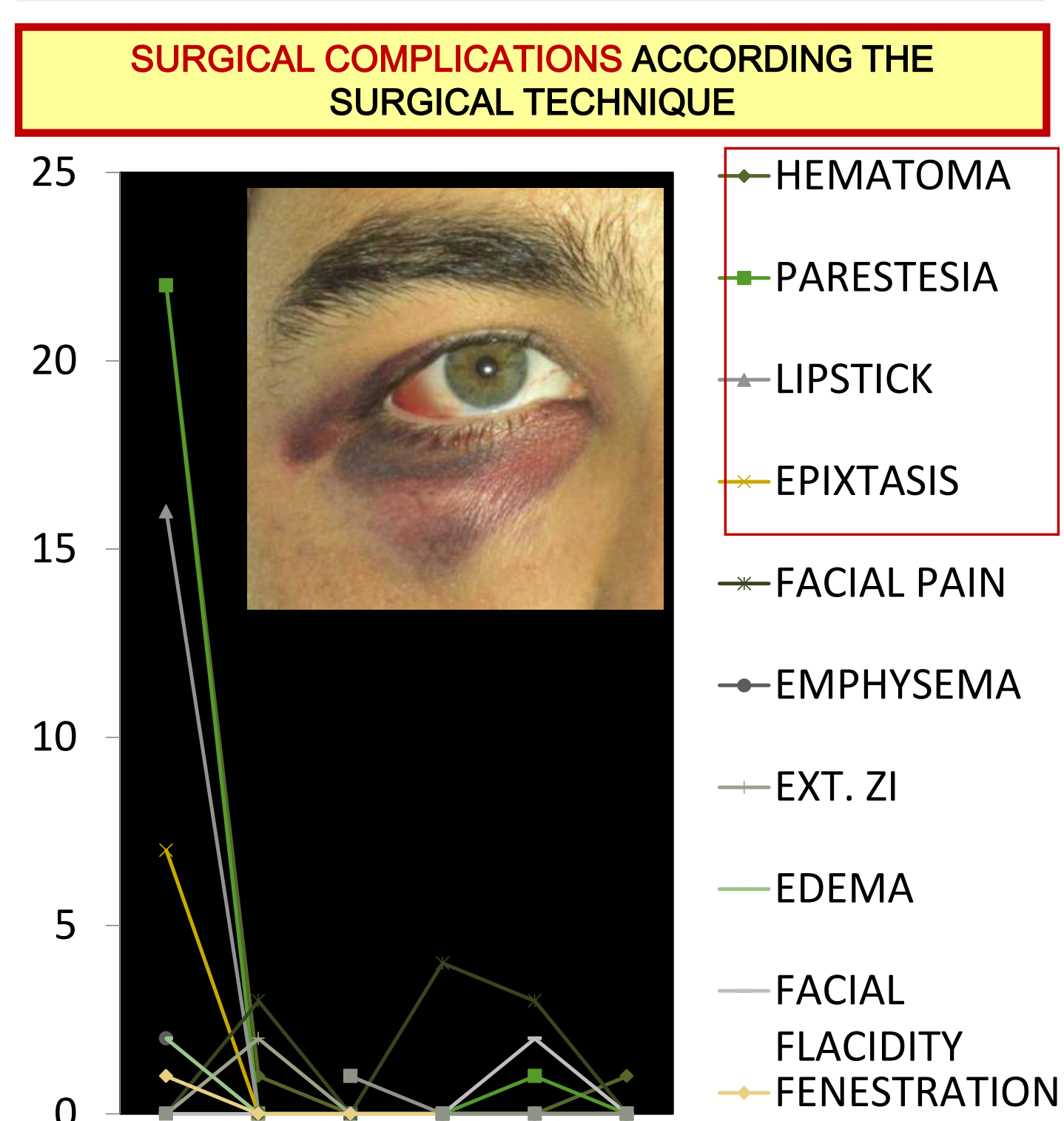

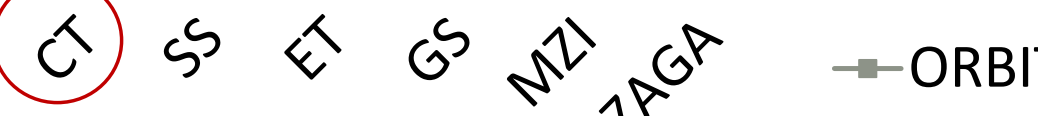
PENETRATION

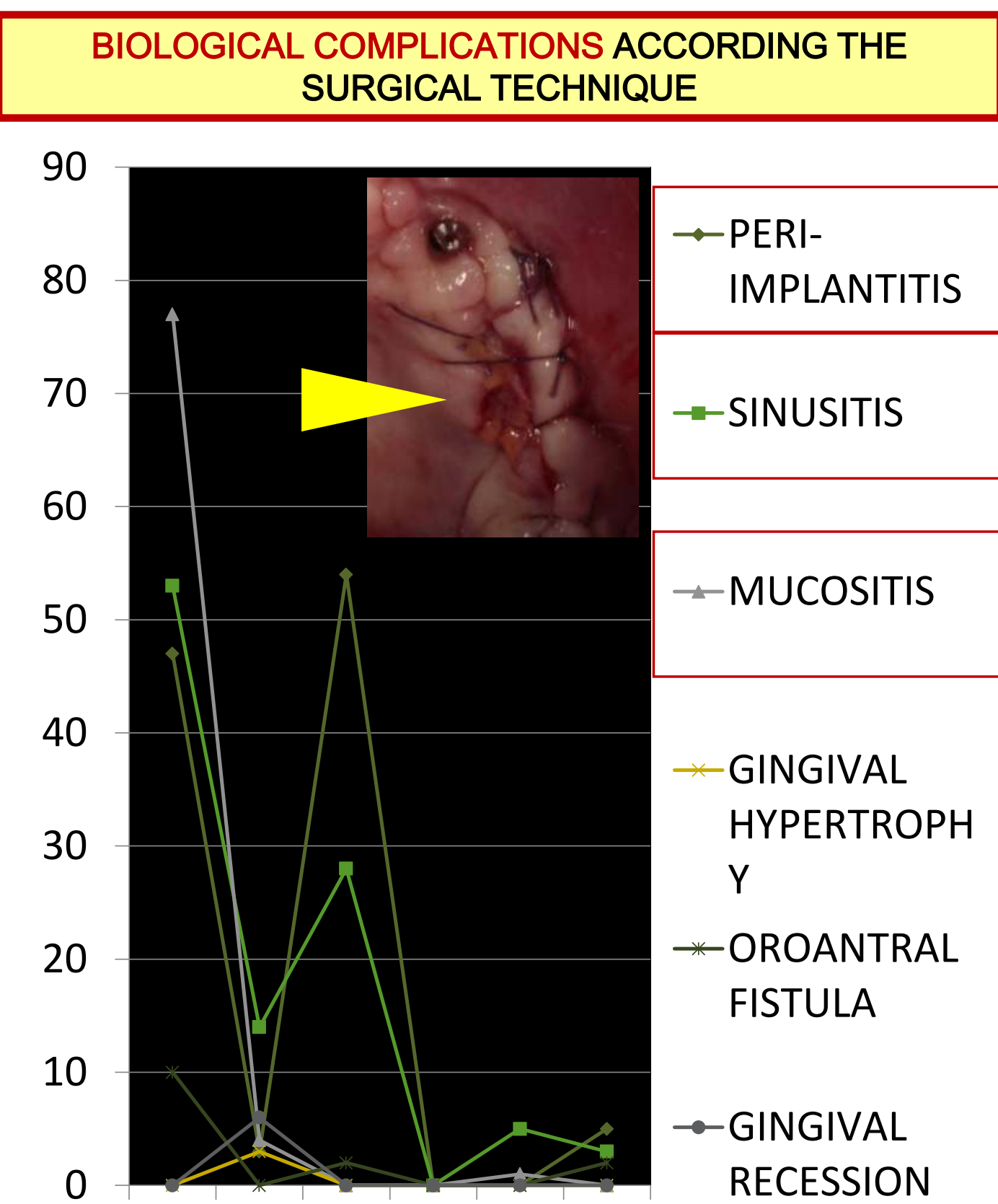

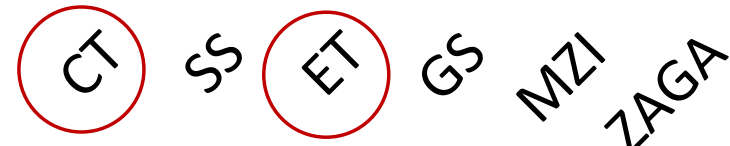

\begin{tabular}{|l|l|l|l|}
\hline \multicolumn{4}{|c|}{ SATISFACTION LEVEL } \\
\hline $\begin{array}{l}\text { Maximum } \\
\text { satisfaction }\end{array}$ & Aesthetic & Function & Phonetic \\
\hline PATIENTS & 354 & 346 & 334 \\
\hline$\%$ & 89,4 & 87,4 & 84,3 \\
\hline
\end{tabular}

Conclusions

The 5-years and 10-years survival rate of Zls was $97.82 \%$ and $95.97 \%$ respectively and it was less with GS.

Immediate loading is the current standard of loading protocol of the Zls. Delayed loading achieves lower survival rate.

The surgical complications depend on the surgical technique used, being higher in the CT.

Mucositis and Sinusits are the most frequent biological complications, more prevalent with CT

Prosthesis fracture is the most frequent prosthetic complication followed by teeth fractures and loosening of screws.

The maximum satisfaction level of patients was $89.4 \%, 87.4 \%$ and $84.34 \%$ for aesthetics,

function and phonetics respectively

- Further studies are needed in the long term.

\section{References}

Goiato MC, et al. J Oral Maxillofac Surg 2014; 43:748-57,
Chrcanovic BR, Abreu MH. Oral Maxillofac Surg 2013; 17: 81-93 Stiévenart M, Malevez C. Int J Oral Maxillofac Surg 2010; 39: 358-63. Schiroli G, Angiero F, Silvestrini-Biavati A, Benedicenti S. J oral Maxillofac Wang F, Monje A, Lin G, Wu Y, M
Maxillofac Impl 2015; 30: 293-8 